\title{
Introduction to Chapter 8
}

Chapter 8 shifts gears, providing a much more theoretical interpretation of ACM processes. In some ways, it takes up where Chapter 1 left off - providing a theoretical update. Whereas Chapter 1 began from the mainly anthropological theories and practices with which we began ACM, Chapter 8 draws on political science, governance, feminist and other more recent social theories to tackle continuing challenges of power asymmetry in community-based natural resources management. The authors emphasis is on understanding the persistence of power imbalances and how ACM can help to shift such imbalances, so common in local resource management institutions.

McDougall and Ojha apply a theoretical lens to their own long-term engagement in Nepal's community forestry to highlight mechanisms underlying the positive shifts in power in that ACM case. They look at three concepts that help to unpack and explain power imbalances: (i) culturally rooted social and gender identities (unmarked categories); (ii) dominant beliefs that are internalized and thus taken-for-granted and unnoticed (doxa); and (iii) political representation (delegation). They then discuss the interplay between structure and agency, an interface that can offer clues to how and when ACM can contribute to changing power relations. In thinking about what actually allows empowerment to occur, they emphasize reflexivity (as emphasized in Chapters 2, 3, 6 and 7) and how it links with deliberative decision making and social learning. They conclude that employing these processes in theoretically grounded ways can greatly enhance ACM's effectiveness in empowerment.

Although this chapter will be more of a struggle for biophysical scientists, given the theoretical orientation and social science language, it provides a very useful understanding of processes that have not been examined in this way or in this detail before. With growing global recognition of the importance of more successful collaborations with communities in a variety of fields - REDD+, forest restoration, climate change mitigation and adaptation, etc. - this chapter provides some excellent theoretical building blocks from which to work. 